\title{
EQUIVALENT CIRCUIT OF RADIATING LONGITUDINAL SLOTS IN SUBSTRATE INTEGRATED WAVEGUIDE
}

HSIN-CHIA LU* AND TAH-HSIUNG CHU

Department of Electrical Engineering

National Taiwan University, Taipei, TAIWAN, R. O. C.

E-mail: leonardo@ew.ee.ntu.edu.tw, the@ew.ee.ntu.edu.tw

This paper presents the equivalent circuit of radiating longitudinal slots in substrate integrated waveguide (SIW). The simulated results using MoM simulator are compared with measured results. They are shown in good agreement. These equivalent circuit will later be used in the design of array antenna using these slots as radiating elements.

\section{Introduction}

The slot antenna array cut on air filled rectangular waveguide is widely used. Since accurate design equation and procedures are established [1-3], an array with large element can be designed without much difficulties. However, the manufacturing cost is high as compared with other platiar antennas such as patch antennas. Integration with other planar circuit and active device are also issues that limit waveguide slot antenna to specific applications.

The spacing between each radiation slot is usually choose to be half wavelength in air-filled waveguide. The spacing is determined solely by the waveguide dimension and is too long if large scan degree is required for the array. The slots on dielectricfilled rectangular waveguide is a way to reduce the distance between radiation elements. In [4], the equivalent citcuit of radiating slots is computed by FDTD.

Since the current at the side wall of TE10 mode rectangular waveguide is in the vertical direction only, a linear array of vias can provide a good path for side wall current. The laminated or substrate integrated waveguide (SIW) proposed at [5-6] provides a good method to produce rectangular waveguides in conventional PCB process or LTCC processes. Since there are natural dielectric filling in these waveguides, the element spacing can also be reduced. By using these process, the cost of manufacturing can be reduced and integration with other planar circuit and active device is easy. A slot array antenna using SIW is presented at [7].

In the design of planar array antenna, the microstrip line has quite a large loss. Since SIW has a lower loss as compared with microstrip line when same substrate is used, In [8], SIW instead of mictostrip line is used to feed each patch element using LTCC process. Due to the high dielectric constant in LTCC, additional vias are placed around the patch antenna to prevent wave propagation along the substrate direction. Since SIW has a clear benefit over microstrip line in the feed structure, it would be nature to use slot antenna on SIW instead of patch antenna as radiating element. Before the array can be designed, the equivalent circuit of a single radiating slot is required. The slot antennas on SIW with different offset are fabricated on Rogers RO4003 substrate. A double taper microstrip line to waveguide transition modified from [9] is used for probing. The same circuits are also simulated by an MoM simulator, the Agilent Momentum for comparison with measured results

The equivalent model of longitudinal radiating slot and equations to calculate its equivalent circuits from scattering parameters are given in Sec. 2 . The measurement and simulation procedure and results are given in Sec. 3. And finally, the conclusion in Sec. 4 . 


\section{Equivalent model of radiating slots}

Large arrays with hundred of slots can be designed in an efficient manner using Elliot's method of design [1-3]. This method is based on that the scattering from the slots on the waveguide wall can be model as a shunt or series equivalent circuits depending on the direction and location of the slot. For a longitudinal slot, it can be modelled as a shunt equivalent circuit as shown in Fig. 1. The scattering parameters at the reference plane that is located at the centre of the slot are related to the shunt equivalent circuit as

$$
\text { . } S_{11}=S_{22}=-\frac{Y / G_{0}}{2+Y / G_{0}} \text { and } S_{21}=S_{12}=1+S_{11}
$$

and $G_{0}$ is the characteristic admittance of the waveguide. With measured scattering parameters, the normalized admittance can be calculated using

$$
\frac{Y(x, l, f)}{G_{0}}=2\left(\frac{1}{S_{11}}-1\right)
$$

Similar expression that use both $S 11$ and $S_{21}$ can be obtained. The admittance of a slot depends on its offset $x$, length $/$ and frequency. The admittance of can be expressed as the following for easy observation of its behaviour versus these variables[10],

$$
\frac{Y(x, y)}{G_{0}}=\frac{G_{r}}{G_{0}} \frac{G+j B}{G_{r}}=g(x) h(y)=g(x)\left[h_{1}(y)+j h_{2}(y)\right]
$$

where $x$ is the offset of the slot, $l$ is the slot length, $g(x)=G_{r}(x) / G_{0}$ is the normalized resonant conductance, $y=l / l_{r}(x, f)$ is the ratio of length to resonant length, $l_{r}(x, f)=\lambda / 2 \pi v(x)$ is the resonant length. $h(y)=\left[h_{1}(y)+j h_{2}(y)\right]=\frac{G+j B}{G_{r}}$ is the ratio of the slot admittance to the resonant conductance.

When the waveguide has dielectric filling, its resonant slot length can be estimated by $\bar{l}=\lambda_{0} / \sqrt{2\left(\varepsilon_{r}+1\right)}$

\section{Results from measurement and simulation}

3.1 Measurement and simulation procedures

The slot antennas on SIW with different offsets are fabricated on Rogers RO4003 substrate. The dielectric constant is 3.38 with substrate thickness of 8 mil and $17 \mu \mathrm{m}$ thick metal. The distance between two linear arrays of vias is 150 mil which will be used as the waveguide width later. The diameter of each via is 12 mil and the distance between each via is also 12 mil. The initial guess of slot length at $30 \mathrm{GHz}$ using (4) is 130 mil. The slot width is 10 mil. Seven slot antennas with different offset starting from $5 \mathrm{mil}$ to $35 \mathrm{mil}$ is fabricated. Double taper microstrip line to waveguide transitions modified from [9] is connected at both ports of waveguide to conver waveguide to microstrip line. The microstrip line is used for input and output ports because two GSG probes and a Cascade probe station are used to probe the circuit for scattering matrix measurement. A set of TRL calibration set that move the reference plane to the middle of the waveguide section is also fabricated. After the reference plane is move into the desired reference plane using TRL calibration method scattering parameters are used to calculate the equivalent circuit of radiating slot using (2). In the meanwhile, scattering matrices of all circuits (including circuits in the TRI calibration kit) between microstrip ports are simulated using Agilent Momentum. The simulated results are processed in the same way as measured scattering matrices. 


\subsection{Results}

The real part and imaginary part of the equivalent circuit of a radiating slot that is 130 mil long, $10 \mathrm{mil}$ width and with 10 mil offset are show in Fig. 2 . Both the measured and simulated results are given. They are shown to be in good agreement. The resonant frequency can be clearly identified as $32.5 \mathrm{GHz}$ where the imaginary part is zero. The resonant frequency is a little depart from $30 \mathrm{GHz}$. The resonant slot admittance versus the ratio of slot length $l$ to resonant length $l r$ is given in Fig. 3(a) and $3(b)$. Compared with the slot admittance given in [4], we found the admittance exhibits a stronger dependence on the slot length which means the bandwidth of our slot antenna is smaller. This is due to that the dielectric constant of our substrate (3.38) is higher than the dielectric constant (2.1) in [4]. The variation of normalized resonant conductance and resonant length versus normalized slot offset (which is $\mathrm{x}$ divided by waveguide width) is given in Fig. 3(c) and 3(d). They also have a stronger dependence on the offset as compared with corresponding values in [4]. Also please note that normalized resonant conductance started to decrease when normalized slot offset is larger than 0.1. This may due to that when the slot is cut in the broad wall of SIW, the current distribution deviated from the original TE10 mode. As the slot offset is increased, the deviation may be larger. The current in the narrow wall will no longer in the vertical direction only. This will then make the variation of admittance different from that of standard metal rectangular waveguide.

\section{Conclusions}

In this paper, we presented the equivalent circuits of a longitudinal radiating slot on the broad wall of SIW. These equivalent circuits are obtained from both measurement results and simulation. Results agreed well in calculated equivalent circuit values. These data will later be used in the design of planar array antenna using these slots as radiating elements.

\section{Acknowledgements}

This paper is supported by the Ministry of Education of R.O.C. under the Grant 89E-FA06-2-4-6.

References

[1] R. S. Elliott, Antenna Theory and Design, Englewood Cliffs, NJ,, Prentice-Hall, 1981, pp.397-423.

[2] R. S. Elliott, "An improved design procedure for small slot arrays," IEEE Trans. Antennas and Propagation, vol. AP-31, pp. 48-53, Jan. 1983.

[3] G. J. Stern and R. S. Elliott, "Resonant length of longitudinal slots and validity of circuit representation: Theory and experiment," IEEE Trans. Antennos and Propagation, vol. AP33, pp. 1264-1271, Nov. 1985

[4] R. V. Gatti, R. Sorentino and M. Dionigi, "Equivalent circuit of radiating longitudinal slots in dielectric filled rectangular waveguides obtained with FDTD method," 2002 IEEE MTT-S Int. Microwave Symp. Dig. , vol. 4, pp 871-874 June 2003.

[5] H. Uchimura, T. Takenoshita, and M. Fujii, "Development of a laminated waveguide," IEEE Trans. Microwave Theory Tech., vol. 46, pp. 2438-2443, Dec. 1998.

[6] H. Uchimura and T. Takenoshita, "Witing board equipped with a line for transmitting a high frequency signal," U.S. Patent 5,982,256, Nov, 9, 1999.

[7] R. V. Gatti, R. Sorentino and M. Dionigi, "Low cost active scanning antenna for mobile satelite terminals," 2003 IEEE AP+S. Dig., vol. 2, pp 680-683 June 2003.

[8] Yong Huang and Ke-Li Wu, "An LTCC array antenna for MM-wave application," 2003 APMC (Asia-Pacific Microwave Conference) dig., vol. 3, pp. 1616-1619. Nov. 2003

[9] D. Deslandes and Ke Wu, "Integrated microstrip and rectangular waveguide in planas form," IEEE Microwave Wireless Comp. Lett., vol. 11, pp. 68-70, Feb. 2001.

[10] R. J. Stegen, "Longitudinal shunt slot characteristics," Hughes Tech. Memo. 261, Hughes Aircraft Co., Culver City, CA, Nov. 1951. (Design curves repreinted in [1], pp. 408-410) 


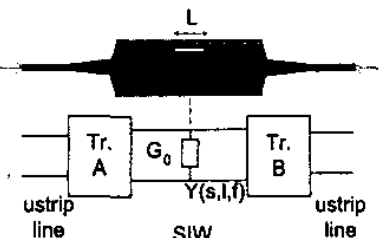

Fig.1 Longitudinal slot in the broad side wall of substrate integrated waveguide (SIW) and its equivalent circuit. Two double taper microstrip line to waveguide transitions are also shown.

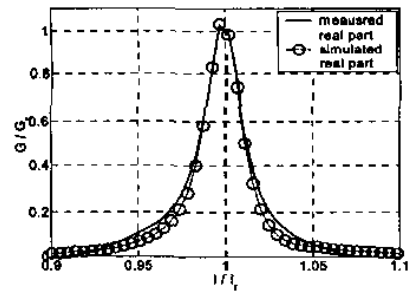

(a)

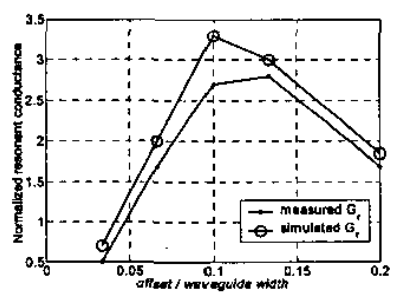

(c)

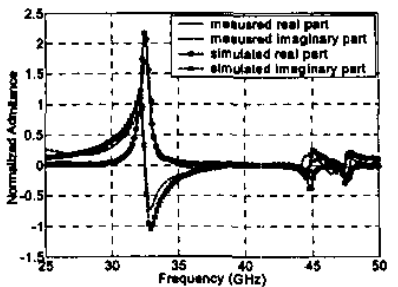

Fig. 2. The real part and imaginary parts of the equivalent circuit of a $130 \mathrm{mil}$ long radiating slot with 10 mil offset. Both measured and simulated results are given.

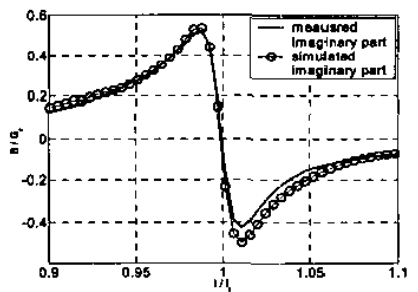

(b)

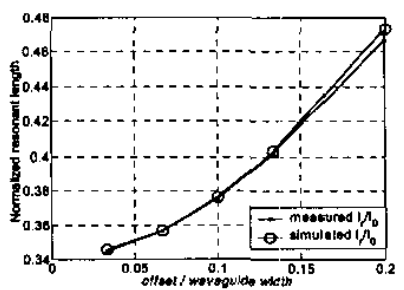

(d)

Fig. 3. (a) $\mathrm{G} / \mathrm{Gr}$ versus $l / l r$, (b) $\mathrm{B} / \mathrm{Gr}$ versus $l / r$ (c) normalized resonant conductance versus normalized offset (d) normalized resonant length versus normalized offset for a longitudinal slot in $\mathrm{SIW}$ at $30 \mathrm{GHz}$ 